mutations. Onset is with visual failure due to retinal degeneration, noted between the ages of 5 and 8 years. This is followed by epilepsy, psychomotor deterioration, sleep disturbance, and extrapyramidal symptoms. Affected females have acne, hirsutism, and obesity. Death follows in the early twenties.

\title{
EARLY DIAGNOSIS OF EPILEPSY SYNDROMES
}

The classification of epilepsy syndromes made initially on the basis of information at time of diagnosis was compared to that made 2 years later in a cohort of 613 children, followed by participating physicians in Connecticut, between 1993 and 1997. After 2 years, syndrome classifications were the same in $86 \%$ of the cohort. The diagnosis was changed in 10\% (mainly incomplete syndromes), and syndrome evolution, mainly West to Lennox-Gastaut, occurred in $4 \%$. Significant changes were rare. (Berg AT, Shinnar S, Levy SR et al. How well can epilepsy syndromes be identified at diagnosis? A reassment 2 years after initial diagnosis. Epilepsia October 2000;41:1269-1275). (Reprints: Dr Anne T Berg, Department of Biological Sciences, Northern Illinois University, DeKalb, IL 60115).

COMMENT. The identification of epileptic syndromes, for the most part, may be made accurately at the time of the initial presentation and diagnosis. Changes in diagnosis at follow-up, necessary in only 14\%, are explained by difficulties in classification of incomplete syndromes and the evolution of West to LennoxGastaut syndomes with age and maturation.

Epileptic syndromes posing problems in diagnosis. Hirsch E et al (Strasbourg, France) review the heterogeneous nature and clinical management of partial epilepsies and incomplete syndromes. BECTS are the most common idiopathic localization-related epilepsy, and may be triggered by carbamazepine in some cases. Primary reading epilepsy and idiopathic occipital lobe epilepsies with photosensitivity are an overlap of idiopathic localization-related and generalized epilepsies, and respond to sodium valproate. Other variants of idiopathic localization-related epilepsies include autosomal dominant nocturnal frontal lobe epilepsy and benign familial infantile convulsions. AED resistance can be due to errors in diagnostic classification of these epilepsy syndromes. EEGvideo evaluation may be necessary in refractory seizures. (Hirsch E et al. New insights into the clinical management of partial epilepsies. Epilepsia Oct 2000;41(suppl 5);S13-S17).

Post-ictal paralysis in BECTS. Dai A et al (State University of New York, Buffalo, NY) found a $9 \%$ association of post-ictal paresis among 68 children with benign rolandic epilepsy, and $50 \%$ had brief post-ictal aphasia. Todd's paresis and aphasia do not exclude the diagnosis of BREC, and these transient complications are clinically benign. (Abstracts from the Annual Meeting of the AES, Los Angeles, CA, Dec 1-6, 2000. Epilepsia Oct 2000;4, suppl 7:88).

\section{ANOXIC AND VASCULAR DISORDERS}

\section{HYPOTHERMIA TREATMENT FOR NEONATAL ENCEPHALOPATHY}

Treatment with mild whole body hypothermia after birth asphyxia was evaluated in 10 of 16 newborns with EEG burst suppression evidence of a bad prognosis, followed at the Imperial College School of Medicine, London, UK. All infants selected for treatment had convulsions and a severe encephalopathy. Hypothermia was instituted within 6 hours of birth and continued for 48 hours. 